\title{
Noise filtering in solar speckle masking reconstructions
}

\author{
C.R. de Boer \\ Max-Planck-Institut für Aeronomie, Max-Planck-Str. 2, D-37191 Katlenburg-Lindau, Germany \\ Universitäts-Sternwarte Göttingen, Geismarlandstr. 11, D-37083 Göttingen, Germany
}

Received June 20; accepted March 18, 1996

\begin{abstract}
A new method of obtaining a sensitive noise filter for solar speckle masking reconstructions is presented below. This filter separates the true image information from noise most reliably. Its efficiency is demonstrated by some representative examples considering observed and artificial image data which were generated in a computer. The latter set of data also suffered realistic degradations by the influence of seeing and noise taken from suitable observations.
\end{abstract}

Key words: techniques: image processing; interferometry — Sun: general

\section{Introduction}

In recent years, speckle interferometry has become an important tool for the study of the solar surface. While the original method introduced by Labeyrie (1970) only gives corrected power spectra, further developments like e.g. the Knox-Thompson algorithm (Knox \& Thompson 1974) yield real diffraction-limited images. This technique was adapted for solar investigations, among others, by von der Lühe $(1985,1994)$.

Until now the best approach to restore seeing-degraded images by speckle methods is the so-called speckle masking algorithm introduced by Weigelt and collaborators (Weigelt 1977; Weigelt \& Wirnitzer 1983; Lohmann et al. 1983). It was proposed for solar application by Pehlemann \& von der Lühe (1989) and employed for the first time by de Boer et al. (1992) and de Boer \& Kneer (1992).

A very important problem in the speckle reconstruction process is the separation of signal from noise. While all available image information has to be collected, noise must be suppressed. Only a reliable noise filter yields a good reconstruction. Since the speckle masking process delivers much more information about the phases of the Fourier transformed reconstruction than necessary, this redundancy can be exploited to improve the statistical reliability.

\section{The speckle masking algorithm}

The measured intensity distribution $i(x)$ of a speckle interferogram can be described by the equation

$$
i(x)=o(x) \otimes p(x),
$$

where $x$ is a two-dimensional spatial coordinate, $o(x)$ denotes the true object-intensity distribution, $p(x)$ the instantaneous point spread function of the combined system of atmosphere and telescope, and $\otimes$ stands for the convolution operator. Fourier transformation renders

$$
I(q)=O(q) \cdot P(q),
$$

where $I(q), O(q)$, and $P(q)$ are the Fourier transformed and therefore complex quantities of Eq. (1), and $q$ is the corresponding two-dimensional Fourier coordinate. Classical speckle interferometry deals with series of Fourier transformed speckle interferograms. Averaging the squared moduli on the assumption that the observed object does not alter yields

$$
\left\langle|I(q)|^{2}\right\rangle=|O(q)|^{2} \cdot\left\langle|P(q)|^{2}\right\rangle,
$$

where $\langle\ldots\rangle$ means the arithmetic mean of the series. If the speckle transfer function (STF) $\left\langle|P(q)|^{2}\right\rangle$ is known, the modulus of the Fourier transformed reconstruction $|O(q)|$ can be calculated.

In order to obtain the real reconstruction, the complex Fourier phases which are lost in Eq. (3) have to be determined additionally. They can be found using the speckle masking algorithm which encodes them as phase differences in the speckle masking bispectrum. This method makes it possible to calculate the true average Fourier phases. Combining them with the corrected amplitudes obtained from Eq. (3) yields the complex Fourier transformed speckle reconstruction.

\subsection{The speckle masking bispectrum}

Speckle images usually consist of discrete, equally spaced intensity values called pixels. For a picture of $N \times N$ 
elements the principal discrete Fourier transform $I(i, j)$ also has $N \times N$ elements, where $i$ and $j$ denote integer spatial-frequency indices. For the principal spectrum, they range between $-N / 2$ and $+N / 2-1$. Using this notation, the direct average frequency component lies at the index $i=0$ and $j=0$. With the same Fourier transformed images as in Eq. (3), the speckle masking bispectrum $B S$ is defined as

$$
B S(q, p)=\langle I(q) \cdot I(p) \cdot I(-q-p)\rangle
$$

or in the discrete form

$$
B S(i, j, k, l)=\langle I(i, j) \cdot I(k, l) \cdot I(-i-k,-j-l)\rangle .
$$

Since $I(+N / 2)=I(-N / 2)$, the indices $i, j, k, l, i+k$, and $j+l$ must range between $-N / 2$ and $+N / 2$. This four-dimensional array contains all information about the phases of the Fourier transformed reconstruction.

The size of the bispectrum is very large for realistic image dimensions. For an image of $64 \times 64$ pixel, the corresponding bispectrum needs a RAM size of 128 Mbyte, assuming that the bispectrum elements are stored as 8 byte complex numbers. This exceeds the equipment of most computers. It is therefore unevadable to essentially reduce the size of the bispectrum.

One possible remedy is to use the symmetry properties of the bispectrum which are given by Pehlemann \& von der Lühe (1989). They reduce the size of the nonredundant bispectrum subset to 6.2 Mbyte, but have the disadvantage that this subset is difficult to handle.

It is a simpler method to restrict the indices $k$ and $l$ to values not exceeding a so-called truncation parameter $M$ :

$$
|k|,|l| \leq M \text {. }
$$

This causes a certain loss of information but nevertheless yields reliable reconstructions. Pehlemann \& von der Lühe recommend $M=5$ as the optimal choice. In order to be even more careful, a truncation parameter of $M=10$ was chosen for this investigation.

An additional possibility to reduce the size of the bispectrum in a simple way is to utilize the elementary symmetry property

$$
B S(i, j, k, l)=B S^{*}(-i,-j,-k,-l),
$$

where ${ }^{*}$ denotes complex conjugation. Using this relation, the range of the indices $k$ and $l$ can be restricted to

$$
0 \leq k \leq M, l=0 \text { and }|k| \leq M, 1 \leq l \leq M .
$$

\subsection{Phase recovery}

Since the Fourier phases are encoded as phase differences in the bispectrum, they have to be recovered recursively using the phase closure equation (cf. e.g. Lohmann et al. 1983)

$$
\mathrm{e}^{\mathrm{i} \phi(i+k, j+l)}=\mathrm{e}^{\mathrm{i} \phi(i, j)} \cdot \mathrm{e}^{\mathrm{i} \phi(k, l)} \cdot \mathrm{e}^{-\mathrm{i} \phi(i, j, k, l)},
$$

where $\phi$ is the object phase, i.e. the phase of the finally reconstructed image, and $\Phi$ the bispectrum phase. In order to avoid phase errors due to very small complex numbers, it is better to consider complex phasors which are normalized to 1 . This causes no loss of information because the modulus can be found using Eq. (3). Since $\phi(0,0)=0$, the recursion has to start with the initial values at the spatialfrequency indices $(1,0)$ and $(0,1)$ which can be taken from the direct average of the Fourier transforms. With these two values and the bispectrum all remaining phasors can be calculated using Eq. (9).

During the recovery process an increasing number of different bispectrum elements and previously calculated phasors contribute to the resulting new object phasor. Considering the spatial-frequency index $(m, n)$ with $m=$ $i+k, n=j+l$, there are several permitted combinations of $i, j, k$, and $l$ which fulfil the truncation conditions. Each of these combinations can be entered in Eq. (9) yielding an individual result of the phasor value. The average of all results gives the best estimate of the object phasor.

Following Pehlemann \& von der Lühe, the numbers of these values give the phase number function $P N$ :

$$
P N(m, n)=\operatorname{card}\left(\mathrm{e}^{\mathrm{i} \phi_{\beta}(m, n)}\right), \beta=1,2,3, \ldots,
$$

where the card function gives the number of elements in a set. Based on this quantity, they define another function which they call phase consistency $P C$ :

$$
P C(m, n)=\left|\frac{1}{P N(m, n)} \cdot \sum_{\beta=1}^{P N(m, n)} \mathrm{e}^{\mathrm{i} \phi_{\beta}(m, n)}\right| .
$$

This function can be regarded as a measure for the reliability of each individual phasor. If all phases in Eq. (11) are equal the consistency amounts to 1 . If the phases are distributed randomly the consistency becomes very small. Therefore one can judge each individual new phasor and distinguish between trustworthy ones and those with a poor signal-to-noise ratio.

This piece of information can be used to improve the phase reconstruction process. With the phase consistency of each calculated phasor taken into account, Eq. (9) can be rewritten as follows

$$
\begin{aligned}
& \mathrm{e}^{\mathrm{i} \phi(i+k, j+l)}= \\
& \quad \mathrm{e}^{\mathrm{i} \phi(i, j)} \cdot P C(i, j) \cdot \mathrm{e}^{\mathrm{i} \phi(k, l)} \cdot P C(k, l) \cdot \mathrm{e}^{-\mathrm{i} \Phi(i, j, k, l)},
\end{aligned}
$$

giving those phasors with a bad signal-to-noise ratio a lower weight on the averaging process.

In order to eliminate single phasors which differ significantly from all the others, one may consider the correlation $C(\alpha)$ of all phasors $\mathrm{e}^{\mathrm{i} \phi_{\alpha}}$ contributing to the object phasor at the Fourier coordinate index $(m, n), 1 \leq \alpha \leq$ 
$P N(m, n)$

$$
C(\alpha)=\frac{1}{P N(m, n)} \cdot \sum_{\beta=1}^{P N(m, n)} \frac{\left|\mathrm{e}^{\mathrm{i} \phi_{\beta}}+\mathrm{e}^{\mathrm{i} \phi_{\alpha}}\right|}{2} .
$$

If $C(\alpha)$ drops below the average of all single correlations reduced by a small offset, the phasor $\mathrm{e}^{\mathrm{i} \phi_{\alpha}}$ can be neglected. This offset prevents the neglection of phasors which are very close to the average. During the phase recovery ac-

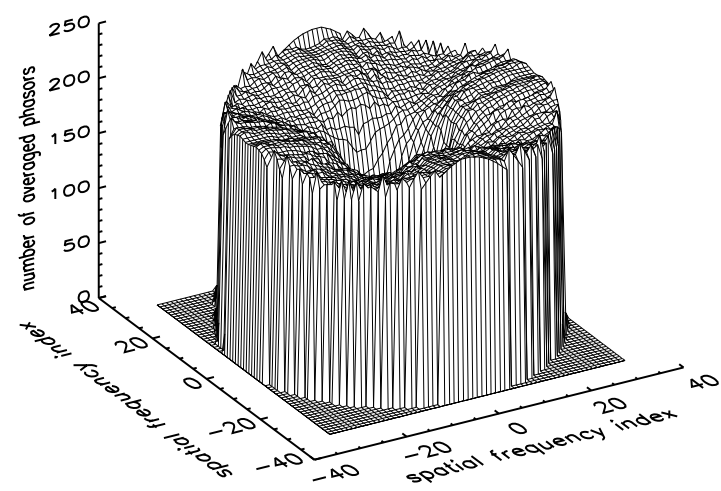

Fig. 1. Phase number function $P N$ of the reconstructions

cording to Eq. (9) or Eq. (12) the succession of the reconstructed frequency indices has to be observed. Phase errors propagate in subsequently calculated phases and might falsify the reconstruction process significantly. Pehlemann \& von der Lühe (1989) propose a sophisticated process to find the next index from the bispectrum which they call Octogon Method.

On the assumption that the power spectrum is radially symmetric and decreases with increasing spatial frequency, there is a simpler way to find the next frequency index. Instead of the four-dimensional bispectrum one may consider the two-dimensional Fourier plane. Here, the next phasor which has to be calculated is the one with the smallest distance from the coordinate origin. This approach guarantees that the points with the highest signalto-noise ratio are calculated first. Therefore, it minimizes the propagation of phase errors.

\section{Treatment of noise}

Every image recording process produces noise. A difficult problem of image reconstruction and especially of speckle interferometry is to separate the image signal from this noise since the latter must not be amplified but should be suppressed. A reliable noise filter is therefore most important. There are several ways to find such a function.

\subsection{General limitation of power amplification}

In order to get the corrected "true" power spectrum, the observed averaged power spectrum has to be divided by the corresponding speckle transfer function (see Eq. (3)). Since no suitable reference as e.g. a star is observable during daylight, this function has to be derived from theory. A good model of the STF has been given by Korff (1973). The only input parameter of his model is the seeing-describing Fried parameter which can be obtained from the spectral ratio method introduced by von der Lühe (1984).

Since the theoretical transfer functions do not consider the influence of noise, they become very small for high spatial frequencies. This means a strong amplification of the power spectrum in this spatial-frequency region. This is also the region where noise becomes dominant. It is therefore advisable to restrict the power amplification to an upper limit and ignore the parts of the power spectrum which would be subjected to a higher amplification. This limit depends on the noise behaviour of the camera system and has to be determined from recorded pictures. In his paper, von der Lühe (1993) claims that the amplitude amplification should only be performed for those parts of the power spectrum for which the STF is larger than $10^{-3}$. The examples in this contribution were calculated with a limit of $10^{-5}$ and with a smoothed transition of the amplification to 0 in the region where the STF drops from $510^{-4}$ to $10^{-5}$ in order to avoid a sharp edge in this function.

\subsection{Noise filter deduced from power information}

Many noise filters are based on the signal-to-noise ratio. Using speckle methods it can be calculated from a comparison between the image power spectrum and the noise power spectrum. The latter can be found from a series of flat-field images. Treating them like ordinary speckle images yields pictures which only contain the residual noise and therefore allow the computation of the noise power spectrum. Considering this two quantities, von der Lühe (1993) developed a noise filter based on the variance of an estimate of the power spectrum that has been corrected for noise. This filter $N F_{1}$ is given by

$$
N F_{1}(q)=\frac{C P(q)-\sigma_{C P}(q)}{C P(q)+\sigma_{C P}(q)}
$$

where $C P(q)$ is the noise-corrected power spectrum, i.e. the image power spectrum reduced by the noise power spectrum, and $\sigma_{C P}$ is the corresponding variance. Since $N F_{1}$ is very noisy and uncertain for high spatial frequencies, von der Lühe recommends some probably empirical truncation and smoothing procedures for his filter.

A simpler but very efficient noise filter giving comparable results is the optimum filter (Brault \& White 1971). 


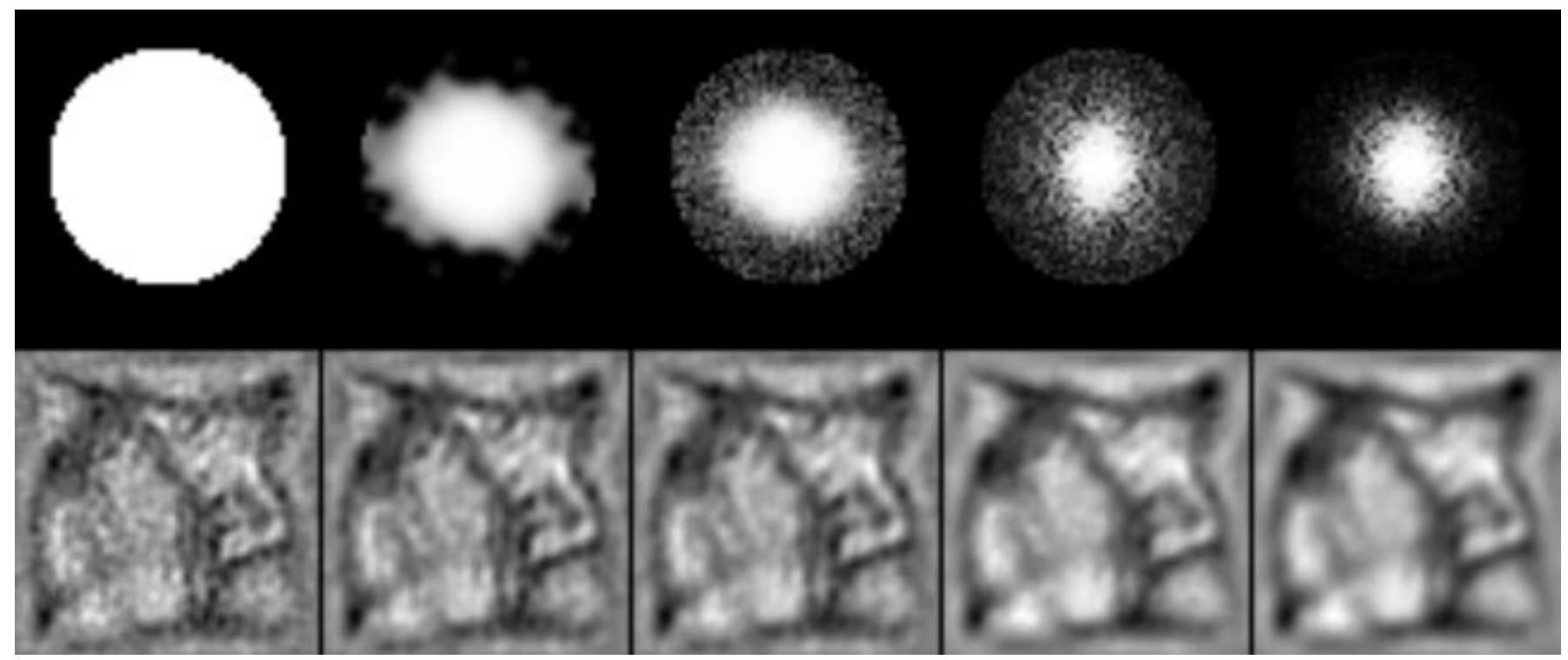

Fig. 2. Speckle reconstructions (lower row) and their corresponding noise filters (upper row); from the left to the right the reconstructions were calculated with the general power limitation, the noise filter of von der Lühe $N F_{1}$, the optimum filter $N F_{2}$, the phase number filter $N F_{3}$, and a combined filter $N F_{2} \cdot N F_{3}$; the grayscale representation of each image was adjusted individually

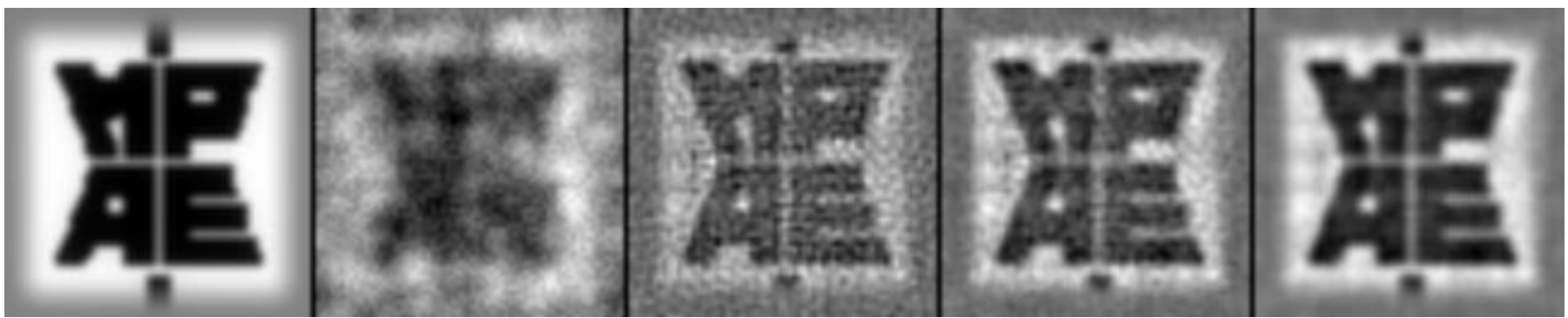

Fig. 3. Test reconstructions of an artificial intensity distribution, from the left to the right this figure represents the original intensity distribution, a typical speckle interferogram affected by seeing and noise, and the reconstructions with the optimum filter $N F_{2}$, the phase number filter $N F_{3}$, and the combined filter $N F_{2} \cdot N F_{3}$; again, each picture is scaled individually

This filter $N F_{2}$ can be written in a slightly modified form as

$$
N F_{2}(q)=\frac{D P(q)-N P(q)}{D P(q)} .
$$

$D P(q)$ represents the averaged image power spectrum and $N P(q)$ the corresponding noise power spectrum. Both filters are close to 1 when the signal-to-noise ratio is large and tend to 0 when the signal-to-noise ratio approaches 1 .

\subsection{Noise filter from phase information}

Another possibility to construct a noise filter function is to evaluate the reliability of each Fourier coordinate index. This quantity is measured by the phase consistency $P C$ given by Eq. (11). As described in the previous section, it quantifies the credibility of each individual phasor in the Fourier plane. The phase consistency is close to 1 for those parts of the Fourier transformed image which have a large signal-to-noise ratio and decreases for less reliable image information. Therefore, it can be used as a third noise filter function $\mathrm{NF}_{3}$

$$
N F_{3}(q)=P C
$$

\section{Examples}

Figure 1 shows the phase number function $P N$ of all following reconstructions which gives the number of averaged phasors for every coordinate index in the Fourier domain.

This figure illustrates that the phase number function increases with increasing spatial frequency until it reaches an upper limit which is caused by the bispectrum truncation. For a truncation parameter of $M=10$ which was chosen for these examples, the maximum of the phase number function amounts to 218 . This high redundancy of 
the phase information is the reason for the high accuracy and reliability of the reconstructions.

In order to test the performance of the different noise filters, speckle masking reconstructions of two different data sets were calculated. The first sample consists of 140 true (i.e. observed) speckle interferograms of solar granulation in a quiet region near the disk centre of a size of $64 \times 64$ pixels corresponding to $5.2 \times 5.2 \operatorname{arcsec}^{2}$ on the Sun. The frames were obtained with the $70 \mathrm{~cm}$ Vacuum Tower Telescope at the Observatorio del Teide in Izaña (Tenerife) at 8:00 UT on August 15, 1993. The seeing conditions were moderate which means a Fried parameter $r_{0} \approx 7 \mathrm{~cm}$. The observed wavelength was $550 \pm 10 \mathrm{~nm}$ and the exposure time $4 \mathrm{~ms}$.

Since finest solar structures are always unknown in detail, the visual judgement of the reconstructions is very difficult. It is hardly possible to decide whether a feature close to the diffraction limit is real or not. In order to dispel this doubt the second set was calculated from theory. An artificial intensity distribution was subjected to all effects of seeing and noise which were extracted from a different series of real speckle interferograms. This procedure yielded a sequence of 140 frames of a size of $64 \times 64$ pixels comparable to the first set. Both series were treated with the same speckle masking procedure.

Figure 2 shows several reconstructions of the first data set using different noise filters. It demonstrates the gain in image quality using more and more sensitive filter functions. The upper row gives the shape of the applied filter in the Fourier domain and the lower row the corresponding reconstructions.

The first picture on the left of Fig. 2 is very noisy since it was calculated only with the general limitation of power amplification. This kind of filter seems to be absolutely insufficient to eliminate the noise content. The next reconstruction to the right was obtained with the noise filter $N F_{1}$ of von der Lühe. Although the image quality is improved it still contains a lot of residual artefacts. The reconstruction based on the optimum filter $N F_{2}$ which is shown in the middle of Fig. 2 nearly gives the same result. Despite a certain improvement there is still some noise present. The next picture to the right was calculated with the phase number filter $N F_{3}$. Compared with the previous images the influence of noise is considerably suppressed. The features have become smooth without losing their fine structure. Finally, the last reconstruction on the right was obtained using a combined filter $N F_{2} \cdot N F_{3}$. This picture appears to be free of noise, but still contains all the details seen in the foregoing reconstructions.

Figure 3 gives the results of the artificial data set. The left image in Fig. 3 represents the original undisturbed intensity distribution before its degradation by seeing and noise. The next picture to the right shows the first frame of the set which is of average quality to give an impression of the influence of seeing and noise. The reconstruction in the centre was calculated with the optimum filter $N F_{2}$, and the next image to the right was obtained with the phase number filter $N F_{3}$. The last picture on the right is based on the combined filter $N F_{2} \cdot N F_{3}$. Again, it is obvious that the image quality increases with increasing consideration of information about the signal-to-noise ratio. Compared with the original image the reconstruction calculated with the combined filter $N F_{2} \cdot N F_{3}$ seems to deliver the best result. It appears to be smooth and free from noise but still to contain all original image information.

\section{Conclusion}

The comparison of the reconstructions obtained with different noise filters proves that the phase consistency is a suitable noise filter function. Best results are obtained when it is multiplied with an optimum filter. The resulting filter function is very sensitive since it considers all power and phase information about the noise in the Fourier domain. Therefore, it is superior to classical noise filters and is highly recommended for solar speckle masking reconstructions.

Acknowledgements. I am grateful to Drs. F. Kneer and E. Wiehr for stimulating discussions and to C. Denker for his assistance during the observations. Thanks are due to Drs. O. von der Lühe and C. Bendlin for helpful comments. This work was supported by the Deutsche Forschungsgemeinschaft through grant Kn $152 / 14-1$. The Vacuum Tower Telescope on Tenerife is operated by the Kiepenheuer-Institut für Sonnenphysik in Freiburg at the Spanish Observatorio del Teide of the Instituto de Astrofísica de Canarias.

\section{References}

Brault J.W., White O.R., 1971, A\&A 13, 169

de Boer C.R., Kneer F., Nesis A., 1992, A\&A 257, L4

de Boer C.R., Kneer F., 1992, A\&A 264, L24

Knox K.T., Thompson B.J., 1974, Astrophys. J. 193, L45

Korff D., 1973, J. Opt. Soc. Am. 63, 971

Labeyrie A., 1970, A\&A 6, 85

Lohmann A.W., Weigelt G.P., Wirnitzer B., 1983, Appl. Opt. 22, 4028

Pehlemann E., von der Lühe O., 1989, A\&A 216, 337

von der Lühe O., 1984, J. Opt. Soc. Am. A1, 510

von der Lühe O., 1985, Speckle - Interferometrische Bildrekonstruktion von Beobachtungen solarer Feinstrukturen, $\mathrm{PhD}$ thesis, Universität Freiburg

von der Lühe O., 1993, A\&A 268, 374

von der Lühe O., 1994, A\&A 281, 889

Weigelt G.P., 1977, Opt. Commun. 21, 55

Weigelt G.P., Wirnitzer B., 1983, Opt. Lett. 8, 389 